\title{
Elementaranalyse der Eihäute von Scyllium stellare, Pristiurus melanostomis und Scyllium canicula und Verteilung des Stick- stoffes in denselben.
}

Von

Hans Buchtala.

(Der Redaktion zugegangen am 10. April 1908.)

Da die Eihäute der Selachier, wie es scheint, nur einmal Gegenstand chemischer Untersuchung waren (Krukenberg), so habe ich auf Veranlassung meines verehrten Lehrers, Herrn Prof. K. B. Hofmann, die Elementaranalyse der oben genannten drei Arten vorgenommen und zugleich die Stickstoffverteilung in denselben bestimmt.

Das Material wurde einige Tage in $0,1 \%$ iger Salzsäure quellen gelassen, hierauf von dem anhaftenden Schleim sowie anderen Verunreinigungen mechanisch gereinigt. Dann wurde die Substanz in Wasser gewaschen, getrocknet und schließlich mit Alkohol und Äther längere Zeit am Rückflußkühler erhitzt. Die Verbrennungen wurden in Pregls automatisch arbeitendem Ofen vorgenommen, der Stickstoff nach Dumas in etwas modifizierter Weise bestimmt und die Schwefelbestimmung wie in meiner früheren Arbeit ${ }^{1}$ ) mit Salpeter und Natriumhydroxyd vorgenommen.

\section{Elementaranalyse.}

I. Scyllium stellare.

Substanz bei $120^{\circ}$ im Vakuum getrocknet. 0,1860 g Substanz gaben 0,3677 g CO$_{2}=\mathrm{C} 53,92 \%$

$0,1219>\mathrm{H}_{2} \mathrm{O}=\mathrm{H} \quad 7,33 \%$

$0,4124 \gg \quad \gg 54,8 \mathrm{ccm} \mathrm{N}=\mathrm{N} 15,08 \%$

bei $729 \mathrm{~mm}$ und $\mathrm{t}=15,5^{\circ} \mathrm{C}$.

0,3300 > $\quad 0,0345 \mathrm{~g} \mathrm{BaSO}_{4}=\mathrm{S} 1,44 \%$

Asche $=0,10 \%$

1) Diese Zeitschrift, Bd. LII, S. 475. 
II. Pristiurus melanostomis.



Zum Vergleiche seien hier auch noch die Analysen vor Scyllium catulus, ${ }^{1}$ ) von der Schalenhaut des Hühnereies $\left.{ }^{2}\right)$ und Coluber natrix ${ }^{3}$ ) beigebracht.

Übersichtstabelle.

\begin{tabular}{|c|c|c|c|c|c|c|}
\hline & $\begin{array}{l}\text { Scyllium } \\
\text { stellare }\end{array}$ & $\begin{array}{l}\text { Pristiurus } \\
\text { melanost. }\end{array}$ & $\begin{array}{l}\text { Scyllium } \\
\text { canicula }\end{array}$ & $\begin{array}{l}\text { Scyllium } \\
\text { catulus }\end{array}$ & $\begin{array}{l}\text { Schalen- } \\
\text { haut des } \\
\text { Hühner- } \\
\text { eies }\end{array}$ & $\begin{array}{c}\text { Coluber } \\
\text { natrix } \\
\text { (Hilger }\end{array}$ \\
\hline C. & 53,92 & 51,45 & 53,64 & 51,50 & 49,78 & 54,68 \\
\hline $\mathrm{H}$ & 7,33 & 6,61 & 6,49 & 6,51 & 6,64 & 7,24 \\
\hline $\mathrm{N}$ & 15,08 & 14,33 & 14,23 & 15,34 & 16,43 & 16,37 \\
\hline $\mathrm{S}$ & 1,44 & 1,52 & 1,33 & 0,88 & 4,25 & $\begin{array}{c}\text { angeblici } \\
\text { S-frei }\end{array}$ \\
\hline
\end{tabular}

Um über die Art der Bindung des Schwefels in den dré untersuchten Objekten einen Aufschluß zu erhalten, wurde de: Versuch gemacht, ob nicht vielleicht ein Teil desselben in

1) Krukenberg, Vergleichend-physiol. Vorträge (Bd. I, S. 226) uni Mitteilungen aus der zoologischen Station zu Neapel (Bd. VI, S. 293 ff., Scyllium catulus Cuv. = Scyllium stellare Günth.

2) Vgl. V. Lindwall, Beitrag zur Kenntnis des Keratins, Upsal: Läkaref. förh. 16.

s) Berliner Berichte, Bd. VI, S. 165. 
Cystin gebunden sei, und ich verarbeitete in dieser Absicht ungefähr je $10 \mathrm{~g}$ der drei Schalenhäute nach der Methode, die ich bei meiner früheren Arbeit ${ }^{1}$ ) angewendet habe.

Scyllium stellare. Der Wasser- und Aschegehalt betrug 10,11\%. Die ammoniakalische Lösung wurde auf $100 \mathrm{ccm}$ ergänzt und davon einmal $10 \mathrm{ccm}$, das zweitemal $20 \mathrm{ccm}$ für die Sehwefelbestimmung verwendet.

Probe I gab 0,0083 $\mathrm{g} \mathrm{BaSO}_{4}$ entsprechend $0,0114 \mathrm{~g} \mathrm{~S}$ in $100 \mathrm{ccm}$. (bezw. $10 \mathrm{~g}$ des Materials).

Probe II gab $0,0142 \mathrm{~g} \mathrm{BaSO}_{4}$ entsprechend $0,0100 \mathrm{~g}$ S in $100 \mathrm{ccm}$.

Pristiurus melanostomis. Hydrolysiert wurden $11 \mathrm{~g}$, die einen Wasser- und Aschegehalt von 15,63\% aufwiesen. Für die Schwefelbestimmung wurden zweimal je $10 \mathrm{ccm}$ der ammoniakalischen Lösung verwendet.

Probe I gab $0,0105 \mathrm{~g} \mathrm{BaSO}_{4}$ entsprechend $0,0144 \mathrm{~g} \mathrm{~S}$ in $100 \mathrm{ccm}$

$>$ II > 0,0113 , > 0,0155 > > > ,

Scyllium canicula. Dieses Material zeigte einen Wasserund Aschegehalt von 15,27\%. Die Schwefelbestimmung wurde zweimal in je $20 \mathrm{ccm}$ gemacht.

Probe I gab 0,0143 g BaSO 4 entsprechend $0,0098 \mathrm{~g} \mathrm{~S}$ in $100 \mathrm{ccm}$

> II , 0,0135 > > $, 0,0093$, > ,

Die obigen Werte von Schwefel berechnen sich auf Cystin folgendermaßen :

Scyllium stellare $=0,44 \%$ Cystin der Eischalen

Pristiurus mel. $=0,60 \%$, ,

Scyllium canic. $=0,42 \%$, ,

Trotz wiederholter Versuche, aus den restierenden ammoniakalischen Lösungen das Cystin in Substanz zu erhalten, ist mir dies nicht gelungen. Es erscheint demnach fraglich, ob der Schwefel überhaupt bei den untersuchten Objekten in einer Cystingruppe gebunden ist, oder ob nur die geringe Menge des etwa vorhandenen Cystins den Mißerfolg erklärt. Die Untersuchung soll, sobald eine größere Menge von Material zur Verfügung steht, wiederholt werden.

1) Diese Zeitschrift, Bd. LII, S. 474. 


\section{Verteilung des Stickstoffes.}

Hierbei wurde die Methode von Hausmann ${ }^{1}$ ) unter Berücksichtigung der Erfahrungen Gümbels ${ }^{2}$ ) angewendet. Es wurden je $4 \mathrm{~g}$ der lufttrockenen Substanz mit $80 \mathrm{~g}$ konzentrierter Salzsäure am Rückflußkühler sechs Stunden lang gekocht. Nach Entfernung der Salzsäure wurde das Ammoniak durch Magnesiumoxyd in Freiheit gesetzt (a). Der Rückstand wurde in Salzsäure gelöst und in einen Meßkolben von $500 \mathrm{ccm}$ Inhalt filtriert. Das Filter mit dem Niederschlag diente zur Bestimmung des Melanin-Stickstoffes (b). Von dem mit Wasser auf $500 \mathrm{ccm}$ ergänzten Filtrate dienten zweimal je $25 \mathrm{ccm}$ zur Bestimmung des Stickstoffes der Mono- und Diaminosäuren (c). Zum Zwecke der Bestimmung des Stickstoffes in den getrennten Mono- und Diaminosäuren wurden zweimal je $100 \mathrm{ccm}$ des

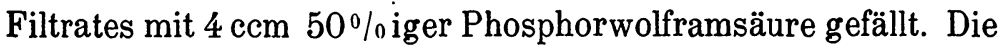
Filtrate von den Phosphorwolframsäureniederschlägen wurden für die Bestimmung des Stickstoffes in den Monoaminosäuren (d), die Niederschläge selbst für die Stickstoffbestimmung in den Diaminosäuren (e) verwendet.

\section{Scyllium stellare.}

Die lufttrockene Substanz hatte einen Aschegehalt von $0,1 \%$ und verlor beim Trocknen auf $120^{\circ} \mathrm{C} .13,98 \%$ Wasser. Ihre Stickstoffmenge betrug 13,75\%.

$0,5642 \mathrm{~g}$ verbrauchten nach $\mathrm{Kjeldahl} 27,55 \mathrm{ccm} \mathrm{n} / 6-\mathrm{HCl}=13,67 \% \mathrm{~N}$ 0,5335 ,

Für die Bestimmung des AmmoniakstickGesamtstickstoff stoffes (a) wurden $10,05 \mathrm{ccm} \quad \mathrm{n} / 3-\mathrm{HCl}$ verbraucht, entsprechend $0,7 \%$ der Probe oder

Zur Auswertung des Melaninstickst. (b) zeigten sich $1,1 \mathrm{ccm} \mathrm{n} / \mathfrak{j}-\mathrm{HCl}$ erforderlich, entsprechend 0,078\% der Probe oder . . . .

Es brauchten $25 \mathrm{ccm}$ des auf $500 \mathrm{ccm}$ ergänzten Filtrates (c) bei zwei Bestimmungen

$5,09 \%$

$0,56 \%$

1) Diese Zeitschrift, Bd. XXVII, S. 95, und Bd. XXIX, S. 136.

2) Hofmeisters Beiträge, Bd. V, S. 297. 
je $9,3 \mathrm{ccm}$ n/5-HCl, was $13,02 \% \mathrm{~N}$ des PräGesamtstickstoff parates oder $94,69 \%$ des Gesamtstickstoffes entspricht.

Die Filtrate mit den Monoaminosäuren (d) verbrauchten $31,3 \mathrm{ccm} \mathrm{n}_{/ 5}-\mathrm{HCl}$, entsprechend 10,96\% der Probe oder . . . . . . .

$79,66 \%$

Die Niederschläge mit den Diaminosäuren (e) verbrauchten $6,2 \mathrm{ccm}$ n/s-HCl, entsprechend 2,17\% der Probe oder

$15,78 \%$

Die Summe der Prozente des in den einzelnen Bestimmungen gefundenen Stickstoffes beträgt sonach

II. Pristiurus melanostomis.

Die lufttrockene Substanz hatte einen Aschegehalt von $0,79 \%$ und zeigte beim Trocknen auf $120^{\circ} \mathrm{C}$. einen Wassergehalt von $12,97 \%$.

Bei der Bestimmung des Gesamtstickstoffes verbrauchten 0,3417 g Substanz 17,80 ccm n/3-HCl, entsprechend $14,59 \% \mathrm{~N}$ des Präparates.

Bei (a) wurden verbraucht $10,70 \mathrm{ccm} \mathrm{HCl,} \mathrm{Gesamtstickstoff}$ entprechend $0,75 \% \mathrm{~N}$ des Präparates oder .

Bei (b) $0,3 \mathrm{ccm} \mathrm{n/5-HCl,} \mathrm{entsprechend}$ 0,021\% des Präparates oder . . . . .

Bei (c) I. $9,9 \mathrm{ccm} \mathrm{n/5-HCl}$ II. 9,7 ,

entsprechend $13,72 \%$ des Präparates oder $94,04 \%$ des Gesamtstickstoffs.

Das Filtrat (d) $27,8 \mathrm{ccm} \mathrm{n/5-HCl,} \mathrm{ent-}$ sprechend 9,70\% des .Präparates oder .

Der Niederschlag (e) $12,0 \mathrm{ccm} \mathrm{n/z-HCl}$. entsprechend 4,20\% des Präparates oder.

$5,13 \%$ $0,14 \%$

$101,09 \%$ 
gefällt und nach gründlichem Waschen und Auskochen des Niederschlages das Filtrat samt den vereinigten Waschwässern auf dem Wasserbade bis zur Krystallisation des Tyrosins eingeengt; die beiden ersten Krystallisationen, die alles Tyrosin enthielten, wurden mit Eisessig ausgekocht, hierauf in Salzsäure gelöst und mit Tierkohle entfärbt. Schließlich wurde das Tyrosin aus der reinen Lösung mit Natriumacetat ausgefällt. Nach zwei Tagen wurde von dem Niederschlage abgesaugt. Die Ausbeute an Tyrosin betrug 1,5 g, entsprechend 6,0\% der untersuchten Probe.

\section{Scyllium canicula.}

Die lufttrockene Substanz hatte einen Aschegehalt von $1,38 \%$ und einen Wassergehalt von 12,31\%.

Bei der Gesamtstickstoff bestimmung verbrauchten $0,5430 \mathrm{~g}$ Substanz 27,8 ccm n/z-HCl, entsprechend 14,34\% N der Probe.

Bei (a) wurden verbraucht $9,2 \mathrm{ccm}^{\mathrm{n}} / 5-\mathrm{HCl}, \quad$ Gesamtstickstoff entsprechend $0,644 \% \mathrm{~N}$ der Substanz oder .

Bei (b) $0,5 \mathrm{ccm} \mathrm{n/5-HCl,} \mathrm{entsprechend}$ $0,035 \% \mathrm{~N}$ der Substanz oder . . . . .

Bei (c) I. und II. $9,8 \mathrm{~cm} \mathrm{n} / 5-H C l$, entsprechend 13,72\% $\mathrm{N}$ der Substanz oder $95,68 \%$ des Gesamtstickstoffs.

Die Filtrate (d) verbrauchten $26,3 \mathrm{ccm}$ $\mathrm{n} /{ }_{5}-\mathrm{HCl}$, entsprechend $9,21 \% \mathrm{~N}$ der Subst. oder

Die Niederschläge (e) verbraucht. $12,6 \mathrm{ccm}$ $\mathrm{n} / 5-\mathrm{HCl}$, entsprechend $4,41 \% \mathrm{~N}$ der Subst. oder

\section{Gesamtstickstoff . .} $4,49 \%$

$0,24 \%$

$64,19 \%$
$30,75 \%$
$99,67 \%$

Auf den Gesamtstickstoff berechnet, gaben Pristiurus mel. und Scyllium can. Zahlenwerte gleicher Ordnung; etwas stärker weicht Scyllium stellare ab durch seinen größeren Gehalt an Melanin- und Monoaminostickstoff und einen kleineren an Diaminostickstoff. Die Stickstoffverteilung bei der Membrana testacea des Huhnes stimmt mit der bei Pristiurus und Scyllium can. gut überein, die.Menge des Diaminostickstoffs nähert sich mehr jener bei Scyllium stellare. 
Elementaranalyse der Eihäute von Scyllium stellare usw.

Zusammenstellung der Resultate.

\begin{tabular}{c|c|c|c|c}
\hline & Ammonik-N & Melanin-N & Monoamino-N & Diamino-N \\
\hline & & & & \\
Scyllium stellare & 0,7 & 0,08 & 10,96 & 2,17 \\
& $(5,09)$ & $(0,56)$ & $(79,66)$ & $(15,78)$ \\
Pristiurus mel. & 0,75 & 0,02 & 9,70 & 4,20 \\
& $(5,13)$ & $(0,14)$ & $(66,45)$ & $(28,78)$ \\
Scyllium canic. & 0,64 & 0,04 & 9,21 & 4,41 \\
& $(4,49)$ & $(0,24)$ & $(64,19)$ & $(30,75)$ \\
Membrana test. & 0,89 & 0,03 & 9,81 & 2,77 \\
des Huhnes & $(6,6)$ & $(0,21)$ & $(72,7)$ & $(20,5)$ \\
$\left(\mathrm{N}=13,6^{\circ} \%\right)$ & & & &
\end{tabular}

Die nicht eingeklammerten Zahlen geben die Prozente des Stickstoffs, bezogen auf die Substanz, an; die in Klammern stehenden bedeuten Prozente des Gesamtstickstoffs. 